\title{
Homopharma: A new concept for exploring the molecular binding mechanisms and drug repurposing
}

\author{
Yi-Yuan Chiu', Jen-Hu Tseng ${ }^{1}$, Kuan-Hsiu Liu', Chih-Ta Lin', Kai-Cheng Hsu', Jinn-Moon Yang 1,2* \\ From Asia Pacific Bioinformatics Network (APBioNet) Thirteenth International Conference on Bioinformatics \\ (InCoB2014) \\ Sydney, Australia. 31 July - 2 August 2014
}

\begin{abstract}
Background: Drugs that simultaneously target multiple proteins often improve efficacy, particularly in the treatment of complex diseases such as cancers and central nervous system disorders. Many approaches have been proposed to identify the potential targets of a drug. Recently, we have introduced Space-Related Pharmamotif (SRPmotif) method to recognize the proteins that share similar binding environments. In addition, compounds with similar topology may bind to similar proteins and have similar protein-compound interactions. However, few studies have focused on exploring the relationships between binding environments and protein-compound interactions, which is important for understanding molecular binding mechanisms and helpful to be used in discovering drug repurposing.
\end{abstract}

Results: In this study, we propose a new concept of "Homopharma", combining similar binding environments and protein-compound interaction profiles, to explore the molecular binding mechanisms and drug repurposing. A Homopharma consists of a set of proteins which have the conserved binding environment and a set of compounds that share similar structures and functional groups. These proteins and compounds present conserved interactions and similar physicochemical properties. Therefore, these compounds are often able to inhibit the proteins in a Homopharma. Our experimental results show that the proteins and compounds in a Homopharma often have similar protein-compound interactions, comprising conserved specific residues and functional sites. Based on the Homopharma concept, we selected four flavonoid derivatives and 32 human protein kinases for enzymatic profiling. Among these 128 bioassays, the $I_{50}$ of 56 and 25 flavonoid-kinase inhibitions are less than 10 $\mu \mathrm{M}$ and $1 \mu \mathrm{M}$, respectively. Furthermore, these experimental results suggest that these flavonoids can be used as anticancer compounds, such as oral and colorectal cancer drugs.

Conclusions: The experimental results show that the Homopharma is useful for identifying key binding environments of proteins and compounds and discovering new inhibitory effects. We believe that the Homopharma concept can have the potential for understanding molecular binding mechanisms and providing new clues for drug development.

\section{Background}

Developing a new drug is difficult and takes on average of 13 years as well as US\$1.8 billion [1]. Traditional approaches for rational drug design used the "one gene,

\footnotetext{
* Correspondence: moon@faculty.nctu.edu.tw

1 Institute of Bioinformatics and Systems Biology, National Chiao Tung University, Hsinchu, 30050, Taiwan

Full list of author information is available at the end of the article
}

one drug, one disease" paradigm to design exquisitely selective ligands of a single disease target. For example, gefitinib (trade name Iressa) and imatinib (trade name Gleevec) have been developed by this strategy and used for lung cancer and chronic myeloid leukemia, respectively. However, many drugs have been indicated that they can interact with more than one target protein [2-4]. Some unexpected efficacy can be linked to activity against 
additional targets, such as imatinib and PDGF receptor [5]. Previous work has identified that a drug interacts with 6 targets on average by analyzing a drug-target network consisting of 802 drugs and 4,746 interactions [6]. Many studies also suggest that drug development on targeting multiple proteins simultaneously can improve efficacy, particularly in the treatment of complex diseases (e.g. cancer and central nervous system disorders) $[7,8]$. The strategy of pharmaceutical research, defined as the specific binding of a compound to two or more molecular targets, has variously been termed "network-based" discovery, "multi-targeted" drug design, "targeted polypharmacology", or "polypharmacology" [9-17].

One challenge of targeted polypharmacology is to identify the molecular targets that bind the compound. To recognize the proteins with similar binding sites of a given protein sequence or structure, the computational approaches of searching the protein sequence or structure databases are usually utilized [18-21]. As the number of protein structures is increasing, protein structures have been proposed to analyze the structural motifs and to describe the binding environments [22,23]. However, most of these studies [24-28] search for similar local structures or binding sites (active sites) based on only one structural motif. Recently, we have introduced Space-Related Pharmamotif (SRPmotif) method [29] to identify pharma-interfaces ( $\geq 2$ structural motifs) from a set of proteins which share similar binding environments. A pharma-interface is consists of a set of spatially discontinuous pharma-motifs which surround the ligand-binding site. In addition, compounds with similar topology would bind to the same or similar proteins and have similar protein-compound interactions $[3,4]$. However, few studies focused on exploring the relationships between binding environments and protein-compound interactions. The atomic interactions between a compound and a protein are important when a compound target a protein. The combination of similar binding environments and protein-compound interactions provides the opportunities to explore the molecular binding mechanisms and is helpful for drug repurposing.

To address this issue, we proposed a new concept of "Homopharma" to describe similar binding environments and the relationships of interactions between proteins and compounds. A Homopharma is a set of proteins which have the conserved sub-binding environment at the protein-compound interfaces and a set of compounds with similar topology. Our results demonstrated that protein-compound complexes of a Homopharma perform similar protein-compound interactions and comprise conserved specific residues and important functional sites. According to the Homopharma concept, four similar flavonoid derivatives were tested against 32 human protein kinases using in vitro enzymatic profiling. The experimental results identified that the $\mathrm{IC}_{50}$ values of 56 and 25 flavonoid-kinase inhibitions are less than $10 \mu \mathrm{M}$ and $1 \mu \mathrm{M}$, respectively. Some novel protein-compound interactions also suggest that these flavonoids could be used as anticancer compounds such as oral and colorectal cancer drugs. These results show that the concept Homopharma is not only useful for identifying potential targets of compounds, but can also reveal the key binding environment. Furthermore, it would be helpful for discovering the new usages for existing drugs. We believe that this approach can be further applied to understand molecular binding mechanisms and take a new direction on drug discovery.

\section{Materials and Methods}

\section{Homopharma and pharma-interfaces}

In this study, we propose a new concept "Homopharma" to describe similar binding environment and the relationships of interactions between proteins and compounds. Proteins sharing similar binding environments can usually be targeted by a set of compound with similar topology. We considered that a Homopharma comprises of a set of proteins which have the conserved sub-binding environment at the protein-compound interfaces and a set of compounds with similar topology. For a complex structure, the proteins with similar pharma-interfaces are first recognized by SRPmotif. The complexes with significant compound topology similarity are reserved. Then, the protein-compound interaction similarity scores are measured. Consequently, the proteins and compounds which share similar protein-compound interactions are considered as a Homopharma. Figure 1 shows the detailed steps to identify a Homopharma from a given complex by the following steps.

\section{Step 1: Search candidates of a pharma-interface}

SRPmotif is utilized to rapidly search potential targets having similar pharma-interfaces (Figure 1A). A pharmainterface can be described as follows: (1) a pharma-interface is a conserved binding interface of multiple proteins which share similar interfaces and are often inhibited by similar compounds; (2) a pharma-interface consists of a set of spatially discontinuous pharma-motifs; (3) a pharma-motif is a short conserved peptide forming a specific sub-interface with interacting residues and specific physico-chemical properties. Each discontinuous pharma-motif of a query complex is translated to 1D sequence with 23 structural alphabets using $\kappa$ and $\alpha$ angles defined in the DSSP program [30] and applied to search against Protein Data Bank (PDB) [31] by 3D-BLAST [32,33]. The candidate structures are then superimposed with the discontinuous pharmamotifs of the query complex by DALI [34], which is a structural alignment tool based on contact similarity patterns. The candidate protein-compound complexes of rootmean-square deviation (RMSD) $\leq 3 \AA$ are reserved. All 
A Search candidates of a pharma-interface

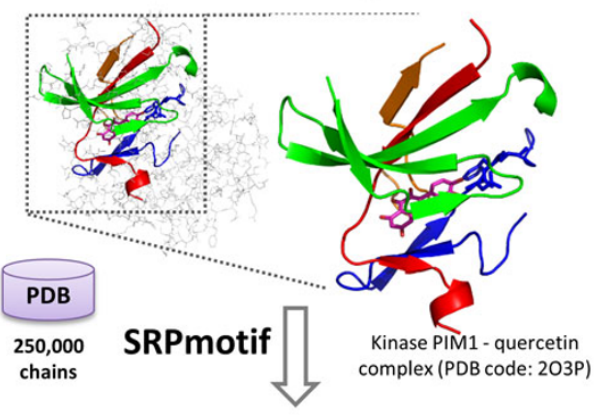

Candidate proteins

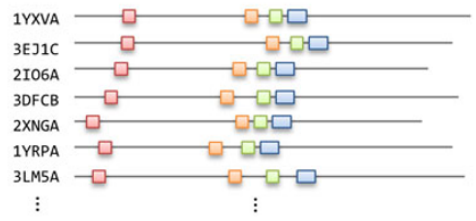

C

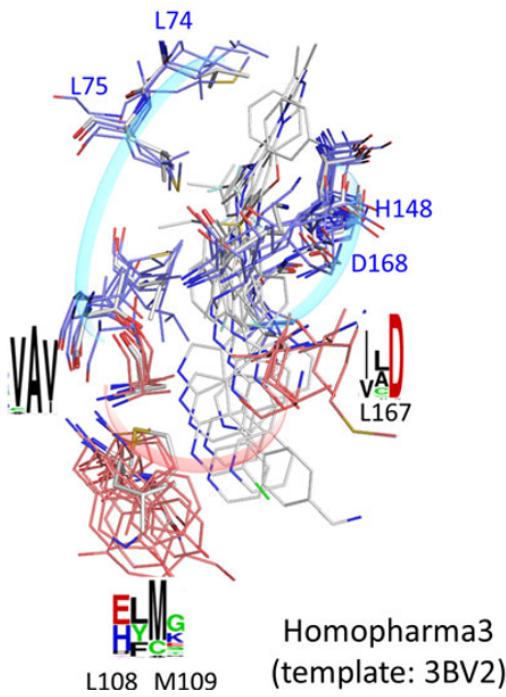

D
B Calculate and cluster interaction profiles

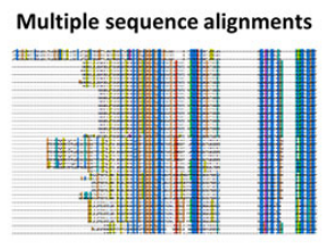

interacting residues
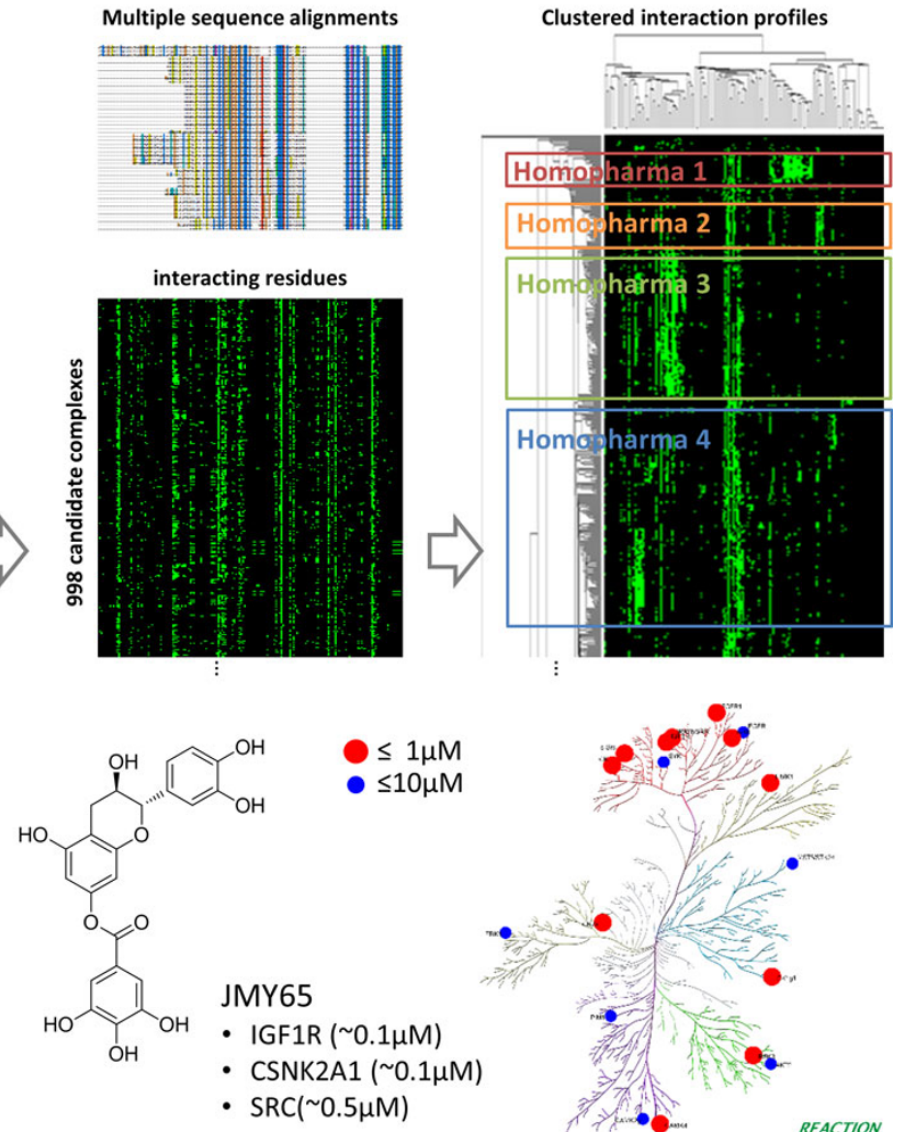

tered interaction profiles

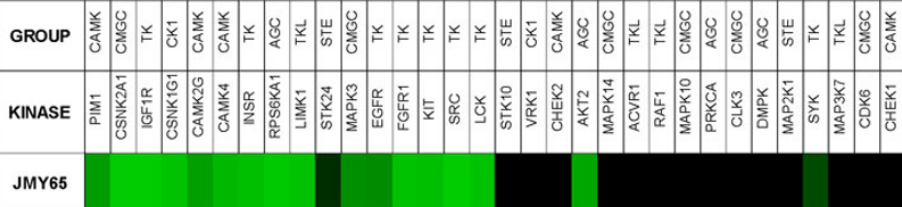

Figure 1 Overview of identification of Homopharma using serine/threonine-protein kinase Pim-1 and quercetin complex as query (A) Search similar pharma-interface candidates of serine/threonine-protein kinase Pim-1 and quercetin complex. (B) The candidates with bound compounds and RMSD $\leq 3 \AA$ are used to generate multiple sequence alignment and the protein-compound interaction profile using iGEMDOCK. The interaction profile is clustered into several Homopharma groups based on interaction similarity scores. (C) Some superimposed complex structures of Homopharma 3. (D) The flavonoid derivative (JMY65) is tested against 32 selected protein kinases based on Homopharma concept. Among 32 bioassays, the $I C_{50}$ of 11 and 18 kinase-JMY65 interactions are less than $1 \mu \mathrm{M}$ (red) and $10 \mu \mathrm{M}$ (blue), respectively. The green and dark denote the inhibition efficiency from high to low.

search parameters of SRPmotif are default. Accordingly, a pharma-interface which consists of several pharma-motifs is constructed based on the conserved binding interfaces. The complexes of the pharma-interface are then used to estimate the protein-compound interaction profile and to evaluate interaction similarities.

\section{Step 2: Calculate protein-compound interaction profiles}

A compound would bind to proteins having similar binding environments; as well as proteins often interact with compounds of similar topology. These proteincompound interactions provide the opportunities to explore the molecular binding mechanisms. After identifications of candidate complexes, similarities between all crystal ligands of complexes are calculated by atom pairs (AP) [35]. The structures whose crystal ligands with significant topology similarity $(\geq 0.6)$ to any compound are reserved. To measure the similarity between protein-compound interactions, a multiple protein 
sequence (or structure) alignment is required. In this work, a multiple protein sequence alignment is utilized and generated by the reserved structures and $T$-coffee [36]. Finally, the protein-compound interaction profile of candidate complexes is calculated based on $i$ GEMDOCK [37]. Different interaction types (electrostatic, hydrogen bonding, and van der Waals) and interactions of main- and side-chain heavy atoms are considered separately.

\section{Step 3: Cluster interaction profiles using interaction similarity}

After estimations of the protein-compound interaction profile, conserved interaction similarity scores between protein-compound interactions are evaluated. To measure the interacting conservation, the conservation weight of each position of multiple sequence alignment (MSA) is given as:

$$
W_{i}^{k}=\frac{f_{i}^{k}}{N}
$$

where $\mathrm{N}$ is the number of compound members of the pharma-interface and $f_{i}^{k}$ is the number of interactions of the residue in MSA position $i$ interacting with compounds with the interaction type $k$ (i.e., electrostatic, hydrogen bonding, and van der Waals). The interaction similarity score between two protein-compound interactions $(A$ and $B)$ is defined as following:

$$
S_{c} A B=\frac{\sum_{i} A_{i} W_{i} \times B_{i} W_{i}}{\sum_{i} A_{i} W_{i}^{2}+\sum_{i} B_{i} W_{i}^{2}-\sum_{i} A_{i} W_{i} \times B_{i} W_{i}}
$$

where $A_{i}$ and $W_{i}$ is the interaction and the conservation weight in position $i$ of the MSA, respectively.

Consequently, the protein-compound interactions are clustered by two-way hierarchical clustering. These interactions are divided into several Homopharma groups, which consist of similar binding environment, compound topology and interactions.

\section{Protein-ligand complex validation dataset}

To verify the Homopharma concept, protein-compound complexes were obtained from PDB. The complexes are eliminated when compounds are metal ions, surface compounds, cofactors, and small compounds (heavy atoms $<6$ ). Two protein-compound pairs are grouped according to the same proteins or compounds. The protein-compound groups are filtered by number of complexes below $50 \%$ of all possible protein-compound combinations. Two groups which overlap with more than $50 \%$ protein or compounds are merged. Finally, 176 protein-compound groups that contain 1,325 complex structures including 672 proteins and 471 compounds are used as validation dataset.

\section{Results and discussion}

\section{Evaluations of compound similarity}

A protein-compound complex having significant compound similarity is considered as a candidate of a Homopharma. In order to decide the threshold of compound topology similarity, we collected 3,151 crystal complex structures of 957 proteins. The distribution demonstrates the relationships between percentage and compound topology similarity (Figure S1A in Additional file 1 ). The precision, recall, and $\mathrm{f}$-measure values were evaluated and the highest F-measure is at 0.36 when the compound topology similarity is 0.6 as well as the precision value is over 0.4. For example, methotrexate (MTX) is the first generation of dihydrofolate reductase (DHFR) inhibitor; the values of compound similarity of COP and DTM to MTX are 0.7 and 0.3 , respectively (Figure S1B in Additional file 1). The compound COP is also the first generation of DHFR inhibitor and has similar topology to MTX; however, DTM is the second generation inhibitor and performs a different binding mode. Another compound estrogen (EST) presents similar results. The compound DES (similarity: 0.8 ) is the estrogen receptor agonist that present the same function with EST, where RAL (similarity: 0.2) is just an estrogen receptor antagonist.

\section{Evaluations of interaction similarity}

To evaluate the performance of interaction similarity, we collected 176 protein-compound groups from PDB. Two protein-compound pairs in the same groups are considered as positive pairs; otherwise, pairs are negative pairs. We also evaluated the performance of identifying protein-compound pairs by protein similarity and compound similarity. The thresholds of protein similarity and compound similarity are BLAST e-value $\leq 10^{-10}$ and topology similarity $\leq 0.6$, respectively. Two pairs are considered as similar interactions while their interaction similarity is greater than 0.6 . The receiver operating characteristic (ROC) curves were estimated to compare protein similarity, compound similarity and interaction similarity (Figure 2). The results showed that interaction similarity (red) provides better performance than protein similarity (blue) and compound similarity (black).

\section{Example analysis: thymidine kinase}

The proteins and compounds in a Homopharma would share conserved interactions and similar physicochemical properties. Therefore, these compounds are often able to inhibit these proteins in a Homopharma. Figure 3A shows that the pharma-interface and Homopharma groups by using the thymidine kinase (TK) of Herpes simplex virus and deoxythymidine (THM) complex (PDB code: $1 \mathrm{KIM}$, chain A) as the query complex. Due to specific expression of herpes thymidine kinase (TK) 


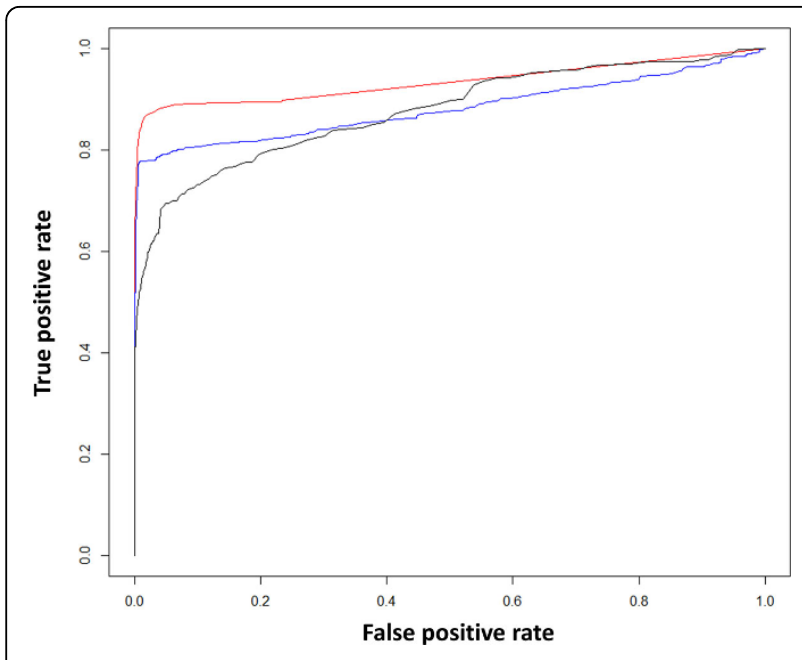

Figure 2 The receiver operating characteristic curves of different similarities. The receiver operating characteristic curves using protein similarity (blue), compound similarity (black), and protein-compound interaction similarity (red) based on 176 proteincompound groups.

in herpesvirus-infection cells, TK becomes an antiviral target of current drugs, such as acyclovir and vidarabine. The identified pharma-interface contains five conserved pharma-motifs. Furthermore, the clustering of interaction profiles demonstrates protein-compound interactions can be separated into four Homopharma groups.

The complexes in a Homopharma present similar binding modes and have the conserved interacting residues (Figures 3B and 3C). Moreover, the conserved interacting residues of these Homopharma groups are often related to ligand binding and catalytic residues. For example, Figure 3B shows the superimposed structures of Homopharma 1 and conserved interacting residues (residue numbering of PDB code: 1OI3). Several interacting residues ( $\mathrm{H} 58, \mathrm{E} 83, \mathrm{~W} 88$, and Y172) are highly conserved in Homopharma 1. Residue E225 is important to form hydrogen bonding that stabilizes the LID region for TK catalytic reaction and H58L/M128F/ Y172F triple mutant has also been indicated that related to the resistance of current anti-TK drugs, such as acyclovir [38]. The conserved interaction profiles are presented in Figure S2A in Additional file 1. However, Homopharma 2 has different conserved interacting residues to Homopharma 1 (Figures $3 \mathrm{C}$ and S2B in Additional file 1). Residues H58, E83, W88, and Y172 are also conserved in Homopharma 1 as well; however, the specific conserved residue R222 of Homopharma 2 interacts with phosphate group of TK substrates [38]. Furthermore, Homopharma 3 and 4 also present different binding environments (Figures S2C and S2D in Additional file 1).

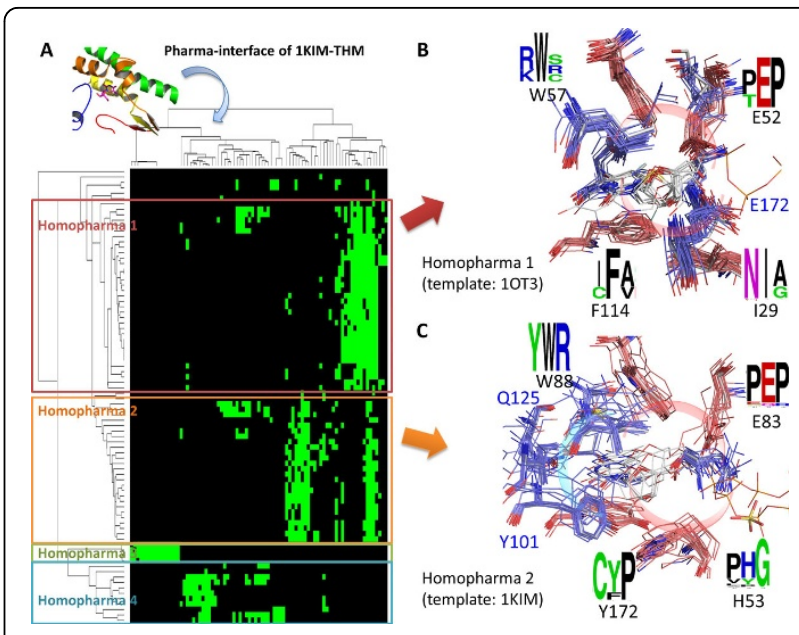

Figure 3 The pharma-interface and Homopharma groups of thymidine kinase and deoxythymidine complex. (A) The pharma-interface of thymidine kinase (TK) and deoxythymidine (THM) complex consists of five pharma-motifs. The proteincompound interactions were grouped into four Homopharma groups based on interaction similarity scores. (B) The superimposed structures of Homopharma 1 and conserved interacting residues (residue numbering of PDB code: 1OI3). (C) The superimposed structures of Homopharma 2 and conserved interacting residues (residue numbering of PDB code: 1KIM).

\section{Example analysis: protein kinases and flavonoid derivatives}

Flavonoids are a class of plant secondary metabolites and have more than 5,000 kinds of derivatives. Flavonoid derivatives have been crystallized within more than 300 protein complex structures, such as protein kinases. Protein kinases play important roles in cell growth and signal transduction $[39,40]$. Protein kinases are one of the most important classes of drug targets, because the deregulation of kinase functions is often implicated in many diseases, such as cancers and neurological and metabolic diseases [40-42]. Although protein kinases share a highly conserved ATP binding environment; however, identical kinase inhibitor has varied inhibitory effects on different kinases [3,4]. Because protein kinases are involved in many complex diseases, many flavonoid derivatives whose biological activities are known on kinases may be repositioned to potential candidates of other diseases.

In this study, we use serine/threonine-protein kinase Pim-1 (PIM1) and quercetin complex (PDB code: 2O3P, chain A) to identify pharma-interface and Homopharma groups. After recognitions of 975 protein-compound complexes by four pharma-motifs, six Homopharma groups are identified according to the interaction similarity (Figures 4A and S3 in Additional file 1). Among these groups, Homopharma 3 shows the highly conserved interactions to DFG motif [43], which is the 


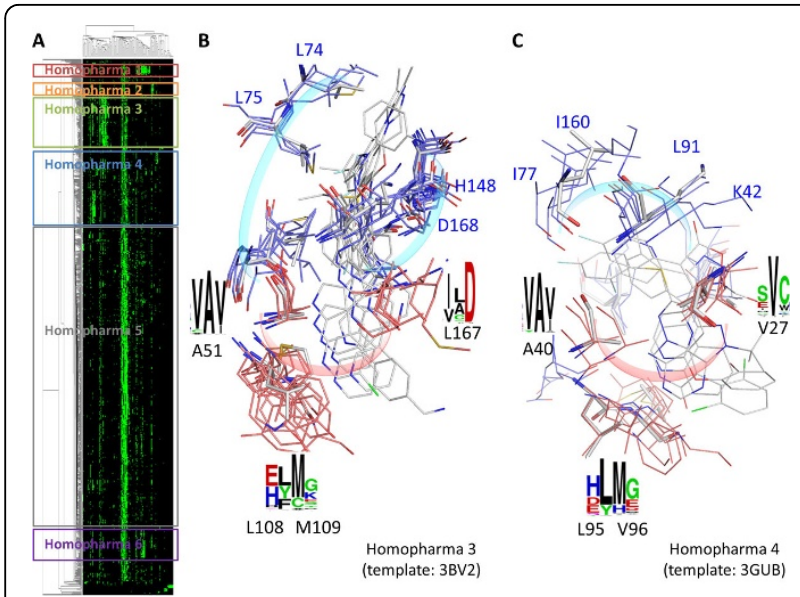

Figure 4 The pharma-interface and Homopharma groups of serine/threonine-protein kinase Pim-1 and quercetin complex. (A) The pharma-interface of serine/threonine-protein kinase Pim-1 (PIM1) and quercetin complex consists of four pharma-motifs. The protein-compound interactions are grouped into six Homopharma groups. (B) The superimposed structures of Homopharma 3 and conserved interacting residues (residue numbering of PDB code: 3BV2). (C) The superimposed structures of Homopharma 4 and conserved interacting residues (residue numbering of PDB code: 3GUB).

beginning of activation loop of kinase (Figure 4B). The conformation of DFG motif is relevant to kinase activation and the conserved aspartic acid binds directly to the magnesium ion cofactor orienting the $\gamma$-phosphate of ATP for transfer. Conserved interacting residues, such as L108 and M109 (residue numbering of PDB code: 3BV2), are involved in hinge motif, which interacts with adenine of ATP. Most compounds of Homopharma 3 belong to Type II kinase inhibitors, which occupy the ATP binding region and allosteric pocket exposed in the kinase inactive conformations $[44,45]$.

Homopharma 4 also performs the conserved interactions to hinge motif (Figure 4C). However, these compounds of Homopharma 4 belong to Type I kinase inhibitors, which target kinases with active conformation and directly compete with ATP binding. Furthermore, compounds of Homopharma 2 are also Type I kinase inhibitors, but have different topology and interacting residues (Figure S3B in Additional file 1). These compounds perform more interactions with residues of $\mathrm{C}$-lobe pocket. Previous study showed that the conserved aspartic acid in C-loop is important in positioning the substrate hydroxyl for in-line nucleophilic attack [46]. Similar observations can be found in other Homopharma groups (Figure S3 in Additional file 1).

\section{Kinase profiling experiment results}

To examine the ability of Homopharma for discovering novel potential targets, we selected quercetin and three similar flavonoid derivatives to test potential target kinases in Homopharma groups. Four selected flavonoid derivatives were obtained from Toronto Research Chemicals (Figure 5). Based on six Homopharma groups of the PIM1-quercetin complex, test kinases were predicted as potential targets by the following step: (1) four flavonoids were docked to these kinase structures of the identified pharma-interface by $i$ GEMDOCK; (2) interaction profiles were analyzed from the docking poses that have the lowest energy; (3) interaction similarity scores were measured to the PIM1-quercetin complex; (4) the kinases of the same Homopharma group have the priority with selection. Most kinases were selected from Homopharma 2, 4, and 5. Furthermore, some kinases which are either not considered potential targets or not searched by pharma-interface were also collected. Eventually, 32 protein kinases were chosen (Table 1 and Figure S4 in Additional file 1).

In vitro enzymatic profiling of the 32 member kinase panel was performed at Reaction Biology Corporation (www.reactionbiology.com, Malvern, PA) using the "HotSpot" assay platform [3]. Each kinase activity assay was performed in duplicate with inhibitor concentration of 10 $\mu \mathrm{M}$ and an ATP concentration of $10 \mu \mathrm{M}$. The experimental results show that three similar flavonoids (quercetin, JMY51, and JMY65) have similar inhibitory effects on the tested 32 kinases. The results indicate that kinases in the same Homopharma group are inhibited by similar compounds. Our results also discover novel interactions, for example, JMY65 has SRC, LCK, and KIT inhibitory activities (Figures 5C and 5E). Moreover, JMY65 could be used to treat oral cancer because of its inhibitory effects on AKT2, IGF1R, EGFR, and MAPK3 (Figures 5C and $5 \mathrm{E}$ ), which are highly expressed in oral cancer cells. Another compound JMY84 could also be used as drug candidates of colorectal cancer due to its effects on CHEK2, LIMK1, and FGFR1 (Figures 5D and 5E).

These 32 tested kinases were divided into two Homopharma groups and the superimposed structures of two groups are presented in Figure 6. Most kinases of Homopharma A are inhibited by tested flavonoid derivatives and have different interacting residues and binding environments than Homopharma B. The locations of DFG motif are varied between these groups (Figure 6A). The co-crystal compounds also suggest the difference of binding environments. These results showed that our approach could discover potential targets of a set of similar compounds based on Homopharma group.

\section{Conclusions}

In this study, we propose a new concept of "Homopharma", combining similar binding environments and protein-compound interaction profiles, to explore the 
A<smiles>O=c1c(O)c(-c2ccc(O)c(O)c2)oc2cc(O)ccc12</smiles>

Quercetin

- $\operatorname{CSNK2A1}(\sim 0.1 \mu \mathrm{M})$

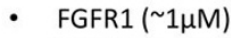

- CAMK2G $(\sim 1 \mu \mathrm{M})$

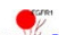

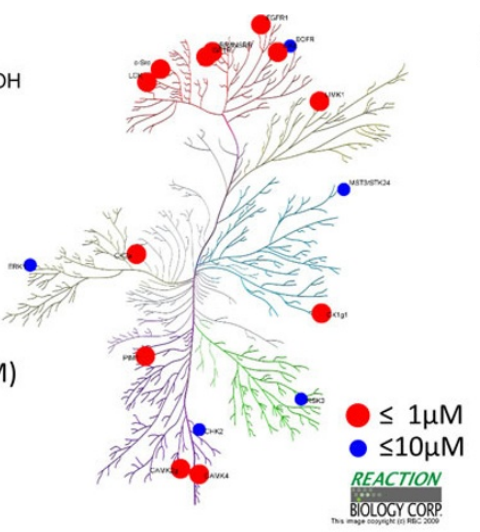

B<smiles></smiles>

JMY51

- $\operatorname{tgF1R}(\sim 1 \mu \mathrm{M})$

- $\operatorname{INSR}(\sim 2 \mu \mathrm{M})$

- fGFR1 $(\sim 3 \mu \mathrm{M})$

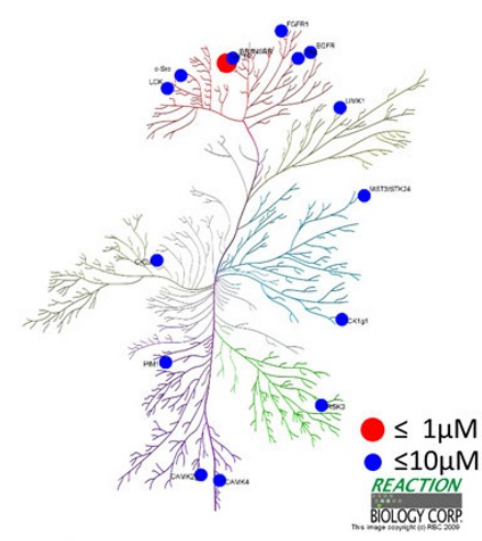

D<smiles></smiles>

JMY84

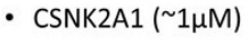

- $\operatorname{IGF1R}(\sim 0.1 \mu \mathrm{M})$

- $\operatorname{CSNK2A1}(\sim 0.1 \mu \mathrm{M})$

- $\quad \operatorname{SRC}(\sim 0.5 \mu \mathrm{M})$

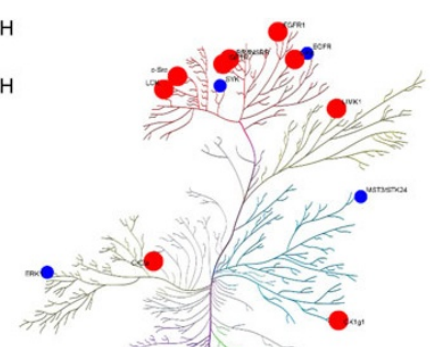

$\mathrm{OH}$
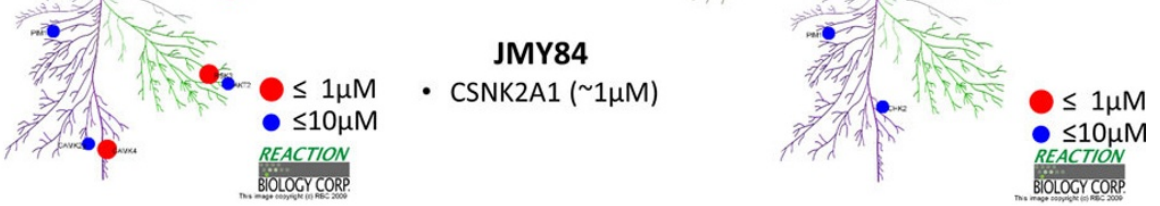

E

\begin{tabular}{|c|c|c|c|c|c|c|c|c|c|c|c|c|c|c|c|c|c|c|c|c|c|c|c|c|}
\hline GROUP & \begin{tabular}{|l|l} 
\\
$\sum_{0}$ & 0 \\
0 & 0 \\
0
\end{tabular} & $\breve{r}$ & 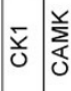 & & $\underline{Y}$ & 遂 & $\overrightarrow{\vec{r}}$ & 岕 & हू & $\stackrel{x}{r}$ & $\check{\vdash}$ & $\check{r}$ & $\leq \underset{\omega}{w}$ & 齐 & $\left.\begin{array}{lll}0 \\
0 \\
0\end{array}\right)$ & $\sum_{0}^{0} \mid \vec{x}$ & $\vec{r}$ & 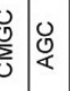 & $\mid \begin{array}{l}0 \\
0 \\
0 \\
0 \\
0\end{array}$ & 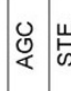 & 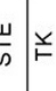 & & 总 & \\
\hline KINASE & 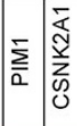 & $\begin{array}{l}\frac{\alpha}{\underline{\underline{U}}} \\
\underline{\underline{\underline{U}}}\end{array}$ & 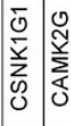 & $\frac{\sum_{0}}{5}$ & 足 & 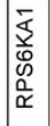 & $\sum_{\underline{\Sigma}}^{\bar{\Sigma}}$ & 壱 & 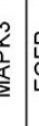 & $\left\{\begin{array}{l}\bar{\alpha} \\
\frac{\bar{u}}{u} \\
u \\
u\end{array}\right.$ & & 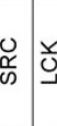 & 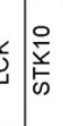 & 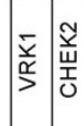 & 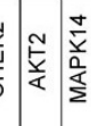 & 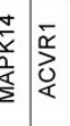 & 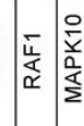 & 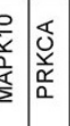 & $\frac{\tilde{2}}{\tilde{U}}$ & 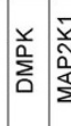 & & & \%ั & \\
\hline \multicolumn{25}{|l|}{ Quercetin } \\
\hline \multicolumn{25}{|l|}{$\begin{array}{l}\text { JMY51 } \\
\text { JMY65 }\end{array}$} \\
\hline JMY65 & & & & & & & & & & & & & & & & & & & & & & & & \\
\hline JMY84 & & & & & & & & & & & & & & & & & & & & & & & & \\
\hline
\end{tabular}

Figure 5 The in vitro enzymatic profiling results. (A) Quercetin and its target kinases. (B) JMY51 and its target kinases. (C) JMY65 and its target kinases. (D) JMY84 and its target kinases. The red and blue points mean the bioactivities are $\leq 1 \mu \mathrm{M}$ and $\leq 10 \mu \mathrm{M}$, respectively. Kinome tree representation is prepared using Kinome Mapper (http://www.reactionbiology.com/apps/kinome/mapper/LaunchKinome.htm). (E) The in vitro enzymatic profiling results of 32 protein kinases and four flavonoid derivatives. The green and dark denote the inhibition efficiency from high to low.

Table 1 List of 32 tested protein kinases for in vitro enzymatic profiling.

\begin{tabular}{cccl}
\hline $\begin{array}{c}\text { Sequence } \\
\text { group }\end{array}$ & $\begin{array}{c}\text { Symbol } \\
\text { name }\end{array}$ & $\begin{array}{c}\text { Kinase SK } \\
\text { number }\end{array}$ & \multicolumn{1}{c}{ Kinase description } \\
\hline AKT2 & AGC & SK019 & v-akt murine thymoma viral oncogene homolog 2 \\
DMPK & AGC & SK111 & dystrophia myotonica-protein kinase \\
PRKCA & AGC & SK303 & protein kinase C, alpha \\
RPS6KA1 & AGC & SK338 & ribosomal protein S6 kinase, 90kDa, polypeptide 1 \\
CAMK2G & CAMK & SK060 & calcium/calmodulin-dependent protein kinase (CaM kinase) II gamma \\
CAMK4 & CAMK & SK061 & calcium/calmodulin-dependent protein kinase IV \\
CHEK1 & CAMK & SK078 & CHK1 checkpoint homolog (S. pombe)
\end{tabular}


Table 1 List of 32 tested protein kinases for in vitro enzymatic profiling. (Continued)

\begin{tabular}{|c|c|c|c|}
\hline CHEK2 & CAMK & SK079 & CHK2 checkpoint homolog (S. pombe) \\
\hline PIM1 & CAMK & SK291 & pim-1 oncogene \\
\hline CSNK1G1 & CK1 & SK647 & casein kinase 1, gamma 1 \\
\hline VRK1 & CK1 & SK389 & vaccinia related kinase 1 \\
\hline CDK6 & CMGC & SK071 & cyclin-dependent kinase 6 \\
\hline CLK3 & CMGC & SK092 & CDC-like kinase 3 \\
\hline CSNK2A1 & CMGC & SK088 & casein kinase 2, alpha 1 polypeptide \\
\hline MAPK10 & CMGC & SK190 & mitogen-activated protein kinase 10 \\
\hline MAPK14 & CMGC & SK264 & mitogen-activated protein kinase 14 \\
\hline MAPK3 & CMGC & SK134 & mitogen-activated protein kinase 3 \\
\hline MAP2K1 & STE & SK217 & mitogen-activated protein kinase kinase 1 \\
\hline STK10 & STE & SK426 & serine/threonine kinase 10 \\
\hline STK24 & STE & SK246 & serine/threonine kinase 24 (STE20 homolog, yeast) \\
\hline EGFR & TK & SK118 & $\begin{array}{l}\text { epidermal growth factor receptor (erythroblastic leukemia viral (v-erb-b) oncogene homolog, } \\
\text { avian) }\end{array}$ \\
\hline FGFR1 & TK & SK143 & fibroblast growth factor receptor 1 (fms-related tyrosine kinase 2, Pfeiffer syndrome) \\
\hline IGF1R & TK & SK174 & insulin-like growth factor 1 receptor \\
\hline INSR & TK & SK178 & insulin receptor \\
\hline KIT & TK & SK201 & v-kit Hardy-Zuckerman 4 feline sarcoma viral oncogene homolog \\
\hline LCK & TK & SK206 & lymphocyte-specific protein tyrosine kinase \\
\hline SRC & TK & SK357 & v-src sarcoma (Schmidt-Ruppin A-2) viral oncogene homolog (avian) \\
\hline SYK & TK & SK363 & spleen tyrosine kinase \\
\hline ACVR1 & TKL & SK026 & activin A receptor, type I \\
\hline LIMK1 & $\mathrm{TKL}$ & SK412 & LIM domain kinase 1 \\
\hline MAP3K7 & $\mathrm{TKL}$ & SK364 & mitogen-activated protein kinase kinase kinase 7 \\
\hline RAF1 & $\mathrm{TKL}$ & SK324 & v-raf-1 murine leukemia viral oncogene homolog 1 \\
\hline
\end{tabular}

molecular binding mechanisms and drug repurposing. A Homopharma consists of a set of proteins sharing conserved binding environment and a set of compounds which have similar structures and functional groups. In

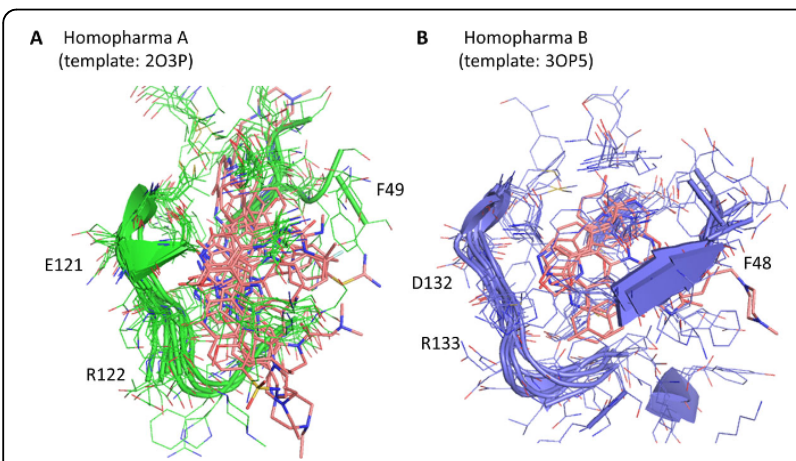

Figure 6 The superimposed structures and enzymatic profiling results. (A) The 16 protein kinases inhibited by four flavonoids (quercetin, JMY51, JMY65, and JMY84) are superimposed (residue numbering of PDB code: 2O3P). Kinases and flavonoids are colored by green and pink, respectively. (B) The structures of 16 protein kinases, which are not inhibited by four flavonoids, are superimposed (residue numbering of PDB code: 30P5). Kinases are colored by blue. a Homopharma, the compounds are often able to inhibit these proteins because their interactions and physicochemical properties are often consensus. Experimental results show that protein-compound complexes of a Homopharma often perform similar interactions in which formed by conserved binding residues (protein sites) and similar important functional groups (compound sites). According to the Homopharma concept, we successfully discovered 56 flavonoid-kinase inhibitions $\left(\mathrm{IC}_{50} \leq 10 \mu \mathrm{M}\right)$ by in vitro enzymatic profiling. Among these 56 inhibitions, the $\mathrm{IC}_{50}$ values of 25 bioassays are less than $1 \mu \mathrm{M}$ and these flavonoids can be considered as potential anticancer compounds. These results show that Homopharma is not only useful to identify potential targets of compounds, but also can reveal the key binding environments. We believe that the Homopharma concept is useful for understanding molecular binding mechanisms and providing new opportunities for drug repurposing.

\section{Additional material}

Additional file 1: Supplementary Figures 


\section{Competing interests}

The authors declare that they have no competing interests.

\section{Authors' contributions}

YYC, CTL, and JMY conceived and designed the experiments. YYC, JHT, KHL, and JMY implemented the materials/analysis programs. YYC, JHT, KHL, CTL, $\mathrm{KCH}$, and JMY performed the experiments and analysed the data. YYC, JHT, $\mathrm{KHL}, \mathrm{CTL}$, and JMY wrote the paper.

\section{Acknowledgements}

This paper was supported by Ministry of Science and Technology, partial supports of Ministry of Education and National Health Research Institutes (NHRI-EX100-10009PI). This paper is also particularly supported by "Aim for the Top University Plan" of the National Chiao Tung University and Ministry of Education. JMY also thanks Core Facility for Protein Structural Analysis supported by National Core Facility Program for Biotechnology.

\section{Declarations}

The publication costs for this paper were funded by the Taiwan Ministry of Science and Technology grant MOST103-2113-M-009-010 awarded to JMY. This article has been published as part of BMC Genomics Volume 15 Supplement 9, 2014: Thirteenth International Conference on Bioinformatics (InCoB2014): Computational Biology. The full contents of the supplement are available online at http://www.biomedcentral.com/bmcgenomics/ supplements/15/S9.

\section{Authors' details}

'Institute of Bioinformatics and Systems Biology, National Chiao Tung University, Hsinchu, 30050, Taiwan. ${ }^{2}$ Department of Biological Science and Technology, National Chiao Tung University, 75 Po-Ai Street, Hsinchu, 30050, Taiwan.

\section{Published: 8 December 2014}

\section{References}

1. Paul SM, Mytelka DS, Dunwiddie CT, Persinger CC, Munos BH, Lindborg SR, Schacht AL: How to improve R\&D productivity: the pharmaceutical industry's grand challenge. Nature Reviews Drug Discovery 2010, 9(3):203-214.

2. Karaman MW, Herrgard S, Treiber DK, Gallant P, Atteridge CE, Campbell BT, Chan KW, Ciceri P, Davis MI, Edeen PT, et al: A quantitative analysis of kinase inhibitor selectivity. Nature Biotechnology 2008, 26(1):127-132

3. Anastassiadis T, Deacon SW, Devarajan K, Ma HC, Peterson JR: Comprehensive assay of kinase catalytic activity reveals features of kinase inhibitor selectivity. Nature Biotechnology 2011, 29(11):1039-1045.

4. Davis MI, Hunt JP, Herrgard S, Ciceri P, Wodicka LM, Pallares G, Hocker M, Treiber DK, Zarrinkar PP: Comprehensive analysis of kinase inhibitor selectivity. Nature Biotechnology 2011, 29(11):1046-1051.

5. Frantz S: Drug discovery: playing dirty. Nature 437(7061):942-943.

6. Mestres J, Gregori-Puigjane E, Valverde S, Sole RV: The topology of drugtarget interaction networks: implicit dependence on drug properties and target families. Molecular Biosystems 2009, 5(9):1051-1057.

7. Roth BL, Sheffler DJ, Kroeze WK: Magic shotguns versus magic bullets: selectively non-selective drugs for mood disorders and schizophrenia. Nature Reviews Drug Discovery 2004, 3(4):353-359.

8. Keith CT, Borisy AA, Stockwell BR: Multicomponent therapeutics for networked systems. Nature Reviews Drug Discovery 2005, 4(1):71-U10.

9. Hopkins AL: Network pharmacology: the next paradigm in drug discovery. Nature Chemical Biology 2008, 4(11):682-690.

10. Zimmermann GR, Lehar J, Keith CT: Multi-target therapeutics: when the whole is greater than the sum of the parts. Drug Discovery Today 2007, 12(1-2):34-42.

11. Csermely P, Agoston V, Pongor S: The efficiency of multi-target drugs: the network approach might help drug design. Trends in Pharmacological Sciences 2005, 26(4):178-182.

12. Korcsmaros T, Szalay MS, Csaba B, Kovacs IA, Csermely P: How to design multi-target drugs: target search options in cellular networks. Expert Opinion on Drug Discovery 2007, 2(6):799-808.
13. Hopkins AL, Mason JS, Overington JP: Can we rationally design promiscuous drugs? Current Opinion in Structural Biology 2006, 16(1):127-136

14. Flordellis CS, Manolis AS, Paris H, Karabinis A: Rethinking target discovery in polygenic diseases. Current Topics in Medicinal Chemistry 2006, 6(16):1791-1798.

15. Dessalew N, Mikre W: On the paradigm shift towards multitarget selective drug design. Current Computer-Aided Drug Design 2008, 4(2):76-90.

16. Keskin O, Gursoy A, Ma B, Nussinov R: Towards drugs targeting multiple proteins in a systems biology approach. Current Topics in Medicinal Chemistry 2007, 7(10):943-951.

17. Morphy R: Selectively Nonselective Kinase Inhibition: Striking the Right Balance. Journal of Medicinal Chemistry 2010, 53(4):1413-1437.

18. Sigrist CJA, Cerutti L, de Castro E, Langendijk-Genevaux PS, Bulliard V, Bairoch A, Hulo N: PROSITE, a protein domain database for functional characterization and annotation. Nucleic Acids Res 2010, 38:D161-D166.

19. Attwood TK, Bradley P, Flower DR, Gaulton A, Maudling N, Mitchell AL, Moulton G, Nordle A, Paine K, Taylor P, et al: PRINTS and its automatic supplement, prePRINTS. Nucleic Acids Res 2003, 31(1):400-402.

20. Pugalenthi G, Suganthan PN, Sowdhamini R, Chakrabarti S: MegaMotifBase: a database of structural motifs in protein families and superfamilies. Nucleic Acids Res 2008, 36:D218-D221.

21. Ku SY, Hu YJ: Structural alphabet motif discovery and a structural motif database. Computers in Biology and Medicine 2012, 42(1):93-105.

22. Weber A, Casini A, Heine A, Kuhn D, Supuran CT, Scozzafava A, Klebe G: Unexpected nanomolar inhibition of carbonic anhydrase by COX-2selective celecoxib: New pharmacological opportunities due to related binding site recognition. Journal of Medicinal Chemistry 2004, 47(3):550-557.

23. Kinnings SL, Liu N, Buchmeier N, Tonge PJ, Xie L, Bourne PE: Drug Discovery Using Chemical Systems Biology: Repositioning the Safe Medicine Comtan to Treat Multi-Drug and Extensively Drug Resistant Tuberculosis. Plos Computational Biology 2009, 5(7).

24. Madsen $D$, Kleywegt $G J$ : Interactive motif and fold recognition in protein structures. J Appl Crystallogr 2002, 35:137-139.

25. Bauer RA, Bourne PE, Formella A, Frommel C, Gille C, Goede A, Guerler A, Hoppe A, Knapp EW, Poschel T, et al: Superimpose: a 3D structural superposition server. Nucleic Acids Res 2008, 36:W47-W54.

26. Debret G, Martel A, Cuniasse P: RASMOT-3D PRO: a 3D motif search webserver. Nucleic Acids Res 2009, 37:W459-W464.

27. Shulman-Peleg A, Shatsky M, Nussinov R, Wolfson HJ: MultiBind and MAPPIS: webservers for multiple alignment of protein 3D-binding sites and their interactions. Nucleic Acids Res 2008, 36:W260-W264.

28. Wu CY, Chen YC, Lim C: A structural-alphabet-based strategy for finding structural motifs across protein families. Nucleic Acids Res 2010, 38(14).

29. Chiu YY, Lin CY, Lin CT, Hsu KC, Chang LZ, Yang JM: Space-related pharma-motifs for fast search of protein binding motifs and polypharmacological targets. Bmc Genomics 2012, 13(Suppl 7):S21.

30. Kabsch W, Sander C: Dictionary of Protein Secondary Structure - PatternRecognition of Hydrogen-Bonded and Geometrical Features. Biopolymers 1983, 22(12):2577-2637.

31. Rose PW, Beran B, Bi CX, Bluhm WF, Dimitropoulos D, Goodsell DS, Prlic A, Quesada M, Quinn GB, Westbrook JD, et al: The RCSB Protein Data Bank: redesigned web site and web services. Nucleic Acids Res 2011, 39: D392-D401.

32. Yang JM, Tung $\mathrm{CH}$ : Protein structure database search and evolutionary classification. Nucleic Acids Res 2006, 34(13):3646-3659.

33. Tung $\mathrm{CH}$, Huang JW, Yang JM: Kappa-alpha plot derived structural alphabet and BLOSUM-like substitution matrix for rapid search of protein structure database. Genome Biology 2007, 8(3).

34. Holm L, Kaariainen S, Wilton C, Plewczynski D: Using Dali for structural comparison of proteins. Current Protocols in Bioinformatic 2006, Chapter 5(Unit 5):5.

35. Carhart RE, Smith DH, Venkataraghavan R: Atom Pairs as MolecularFeatures in Structure Activity Studies - Definition and Applications. Journal of Chemical Information and Computer Sciences 1985, 25(2):64-73.

36. Notredame C, Higgins DG, Heringa J: T-Coffee: A novel method for fast and accurate multiple sequence alignment. J Mol Biol 2000, 302(1):205-217. 
37. Hsu KC, Chen YF, Lin SR, Yang JM: iGEMDOCK: a graphical environment of enhancing GEMDOCK using pharmacological interactions and postscreening analysis. Bmc Bioinformatics 2011, 12.

38. Pilger BD, Perozzo R, Alber F, Wurth C, Folkers G, Scapozza L: Substrate diversity of herpes simplex virus thymidine kinase. Impact Of the kinematics of the enzyme. The Journal of biological chemistry 1999, 274(45):31967-31973.

39. Blume-Jensen P, Hunter T: Oncogenic kinase signalling. Nature 2001, 411(6835):355-365.

40. Cohen P: Protein kinases - the major drug targets of the twenty-first century? Nature Reviews Drug Discovery 2002, 1(4):309-315.

41. Noble MEM, Endicott JA, Johnson LN: Protein kinase inhibitors: Insights into drug design from structure. Science 2004, 303(5665):1800-1805.

42. Weinmann $\mathrm{H}$, Metternich R: Drug discovery process for kinase inhibitors. Chembiochem 2005, 6(3):455-459.

43. Liao JاJL: Molecular recognition of protein kinase binding pockets for design of potent and selective kinase inhibitors. Journal of Medicinal Chemistry 2007, 50(3):409-424

44. Liu Y, Gray NS: Rational design of inhibitors that bind to inactive kinase conformations. Nature Chemical Biology 2006, 2(7):358-364

45. Norman RA, Toader D, Ferguson AD: Structural approaches to obtain kinase selectivity. Trends in Pharmacological Sciences 2012, 33(5):273-278.

46. Zhou J, Adams JA: Is there a catalytic base in the active site of CAMPdependent protein kinase? Biochemistry 1997, 36(10):2977-2984.

\section{doi:10.1186/1471-2164-15-S9-S8}

Cite this article as: Chiu et al:: Homopharma: A new concept for exploring the molecular binding mechanisms and drug repurposing. BMC Genomics 2014 15(Suppl 9):S8.

\section{Submit your next manuscript to BioMed Central and take full advantage of:}

- Convenient online submission

- Thorough peer review

- No space constraints or color figure charges

- Immediate publication on acceptance

- Inclusion in PubMed, CAS, Scopus and Google Scholar

- Research which is freely available for redistribution

Submit your manuscript at www.biomedcentral.com/submit 